\title{
Résumé de thèse. Les dynamiques d'engagement et de désengagement au travail
}

Contribution à leur conceptualisation en clinique du travail, à partir d'une intervention dans un service public de la propreté

\section{Amélie Sandoval}

\section{(QpenEdition}

Journals

Édition électronique

URL : https://journals.openedition.org/activites/7118

DOI : 10.4000/activites.7118

ISSN : $1765-2723$

Éditeur

ARPACT - Association Recherches et Pratiques sur les ACTivités

Référence électronique

Amélie Sandoval, «Résumé de thèse. Les dynamiques d'engagement et de désengagement

au travail », Activités [En ligne], 18-2 | 2021, mis en ligne le 15 octobre 2021, consulté le 07 avril 2022.

URL : http://journals.openedition.org/activites/7118; DOI : https://doi.org/10.4000/activites.7118

Ce document a été généré automatiquement le 7 avril 2022.

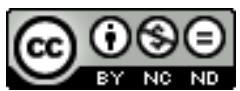

Activités est mis à disposition selon les termes de la licence Creative Commons Attribution - Pas d'Utilisation Commerciale - Pas de Modification 4.0 International. 


\section{Résumé de thèse. Les dynamiques d'engagement et de désengagement au travail}

Contribution à leur conceptualisation en clinique du travail, à partir d'une intervention dans un service public de la propreté

\section{Amélie Sandoval}

\section{RÉFÉRENCE}

Sandoval, A. (2020). “L'engagement et le désengagement au travail. Contribution à leur conceptualisation en clinique du travail, à partir d'une intervention dans un service public de la propreté". Thèse de doctorat, Cnam-Hésam Université, Paris, France. https://tel.archives-ouvertes.fr/tel-03151307

\section{NOTE DE L'ÉDITEUR}

Thèse de doctorat en psychologie.

Soutenue publiquement le 20 mars 2020.

École Doctorale Abbé Grégoire, Le Cnam - HESAM universités.

Centre de recherche sur le travail et le développement (CRTD, EA 4132)

Directrice de thèse :

Katia KOSTULSKI, Professeure des universités en Psychologie, Cnam

Jury de thèse :

Flore BARCELLINI, Professeure des universités en Ergonomie, Cnam (présidente) Brigitte ALMUDEVER, Professeure des universités en Psychologie sociale du travail et des organisations, Université Toulouse Jean Jaurès (rapporteure) Philippe SARNIN, Professeur des universités en Psychologie du travail et des organisations, Université Lumière Lyon 2 (rapporteur) 
Dominique LHUILIER, Professeure émérite des universités en Psychologie du travail, Cnam (examinatrice)

Simon VIVIERS, Professeur agrégé en Sciences de l'orientation, Université Laval Québec (examinateur)

Fernando ANDRADE, Chef du service de la prévention et des conditions de travail, Direction de la Propreté et de l'Eau, Mairie de Paris (invité)

\section{Problématique}

1 Ce travail trouve son origine dans une intervention en clinique de l'activité (Clot, 2008 ; Kostulski, 2010), menée dans un service municipal de la propreté auprès de chefs d'équipe d'éboueurs et de leur ligne hiérarchique. À partir d'une commande du service, cette intervention a consisté à co-analyser avec treize chefs d'équipe du nettoiement des difficultés qu'ils rencontraient dans leur activité, puis à les mettre en discussion entre eux et leur ligne hiérarchique. L'objectif était d'expérimenter des cadres coopératifs permettant de trouver collectivement des ressources face aux obstacles rencontrés par les professionnels, et de développer de nouvelles modalités de travail, entre échelons et fonctions, dans le service.

2 L'intervention et les analyses menées ont à la fois provoqué et mis au jour des mouvements d'engagement et de désengagement chez les professionnels, tant dans l'intervention elle-même que dans leur activité quotidienne. Ce sont ces mouvements que nous avons pris pour objet de notre travail de recherche, en nous interrogeant sur leurs ressorts et sur leurs incidences, dans le travail et pour les professionnels.

\section{Méthodes}

3 Nous avons cherché à spécifier et comprendre les formes d'engagement et de désengagement que nous avons rencontrées, en nous appuyant sur des matériaux empiriques et cliniques issus de notre expérience de terrain, que nous avons mis en discussion avec des apports théoriques de la psychologie du travail et des organisations (Kahn, 1990 ; Meyer \& Allen, 1991; Morrow, 1983; Paillé, 2005; Schaufeli, 2013; Vandenberghe, 2016) et de différentes perspectives en psychopathologie du travail et en clinique du travail (Clot, 2004 ; Dejours, 2008 ; Lhuilier, 2006). Nos matériaux étaient pour leur part essentiellement constitués de dialogues (enregistrés et/ou filmés) issus d'autoconfrontations simples et croisés (27) et de réunions collectives menées au cours de notre intervention (29), ainsi que de notes d'observation et de notre journal de terrain. Le travail de recherche mené a tenté à la fois d'éclairer ces matériaux à partir des théories et de mettre les théories à l'épreuve des matériaux.

\section{Résultats}

4 Cette confrontation entre nos matériaux empiriques et cliniques et différentes ressources théoriques nous a amenés à défendre la pertinence et l'intérêt, à la fois théorique et clinique, d'analyser l'engagement et le désengagement en rapport avec l'activité menée par les professionnels. 
5 Cette prise en compte de l'activité et des situations réelles, en nous appuyant plus particulièrement sur la modélisation de l'activité développée en clinique de l'activité (Clot, 2004, 2008), à partir notamment des travaux de Vygotski $(1997,2017)$ et Leontiev (1984), et la mobilisation de différents travaux en psychopathologie, psychodynamique du travail et psychosociologie du travail (Dejours, 2008; Duarte, 2017; Lhuilier, 2006 ; Molinier, 2010; Roche, 2016; Veil, 2012; Viviers, 2017) ont permis la formulation de différentes propositions théoriques concernant l'engagement et le désengagement au travail :

- L'engagement et le désengagement au travail ne sont pas des états, mais des processus dynamiques.

-L'engagement et le désengagement se développent et s'infléchissent dans le cours de l'activité, dont ils peuvent être des modalités, des sources, et des effets, voire des visées, dans des rapports de co-engendrement.

- L'engagement comme le désengagement peuvent être des ressources ou des obstacles à la santé des professionnels et à l'efficacité de leur travail et de l'organisation (sans forcément qu'il y ait une convergence entre ces différents niveaux).

- L'engagement et le désengagement ne sont pas exclusifs l'un de l'autre, ils peuvent être concomitants et parfois même liés, et sont potentiellement conflictuels ou ambivalents.

6 Une analyse langagière de dialogues issus de l'intervention et des processus psychologiques qui les sous-tendent (Kostulski, 2011), s'appuyant sur des approches pragmatiques de l'énonciation (Rabatel, 2008; Vion, 2005), a permis d'étayer ces différentes propositions en permettant d'identifier concrètement des dynamiques particulières d'engagement et de désengagement (comme par exemple des formes d'engagement empêché ou des formes de désengagement défensif et/ou revendicatif), leurs liens avec l'activité, ainsi que leurs implications subjectives et leurs effets sur le travail.

\section{Thèse défendue}

7 Ce travail nous amène ainsi à proposer de concevoir l'engagement et le désengagement au travail comme des processus dynamiques, qui se développent dans la rencontre entre un sujet et une situation, et relèvent de dimensions à la fois individuelles et organisationnelles, psychologiques et sociales. Ces conduites sont chargées de significations et peuvent prendre différentes formes et fonctions dans l'activité. Elles peuvent permettre de s'adapter, de se défendre et de résister dans les situations vécues, mais aussi de les transformer et d'agir sur soi et son environnement.

\section{Discussion}

8 À partir de là sont discutés les effets possibles du type de dispositif d'intervention que nous avons déployé sur l'engagement et le désengagement, ainsi que les rapports entre engagement, désengagement et santé. Est notamment questionné le caractère potentiellement pathogène ou, à l'inverse, favorable au développement des sujets et de leur activité, des dynamiques d'engagement et de désengagement identifiées. 


\section{BIBLIOGRAPHIE}

Clot, Y. (2004). La fonction psychologique du travail (4e édition corrigée). Paris : Presses universitaires de France.

Clot, Y. (2008). Travail et pouvoir d'agir. Paris : Presses universitaires de France.

Dejours, C. (2008). Travail, usure mentale. Essai de psychopathologie du travail (4 éd.). Paris, France : Bayard.

Duarte, A. (2017). Défenses et résistance en psychodynamique du travail (Thèse de doctorat inédite, Université Paris Descartes, France).

Kahn, W. A. (1990). Psychological conditions of personal engagement and disengagement at work. Academy of Management Journal, 33(4), 692-724. Repéré à : http://dx.doi.org/10.2307/256287

Kostulski, K. (2010). Quelles connaissances pour l'action en clinique du travail ? Une contribution à la question des rapports entre les actions de transformation et la production de connaissances en clinique du travail. In Y. Clot, \& D. Lhuilier (Eds.), Agir en clinique du travail (p. 27-38). Toulouse, France : Érès

Kostulski, K. (2011). Formes et fonctions psychologiques des réalisations langagières : vers une psychologie concrète du langage (HDR, Université Paris 8, Saint-Denis, France).

Leontiev, A. (1975/1984). Activité, conscience, personnalité. Moscou, Russie : Éditions du Progrès.

Lhuilier, D. (2006). Cliniques du travail. Toulouse : Érès.

Molinier, P. (2010). Souffrance, défenses, reconnaissance. Le point de vue du travail. Nouvelle revue de psychosociologie, 10(2), 99-110.

Morrow, P.C. (1983). Concept Redundancy in Organizational Research: The Case of Work Commitment. Management Publications, $\mathrm{n}^{\circ}$ 47, 486-500.

Paillé, P. (2005). Engagement organisationnel et modes d'identification. Dimensions conceptuelle et empirique. Bulletin de psychologie, 58-6(48, 705-711.

Rabatel, A. (2008). Homo narrans. Pour une analyse énonciative et interactionnelle du récit. Limoges, France : Lambert Lucas.

Roche, P. (2016). La puissance d'agir au travail. Recherches et interventions cliniques. Toulouse, France : Érès.

Schaufeli, W.B. (2013). What is engagement? In C. Truss, K. Alfes, R. Delbridge, A. Shantz, \& E. Soane (Eds.), Employee Engagement in Theory and Practice (p. 15-35). London, UK : Routledge.

Vandenberghe, C. (2016). L'engagement organisationnel. In G. Valléry, M.-É. Bobillier Chaumon, É. Brangier, \& M. Dubois. Psychologie du Travail et des Organisations : 110 notions clefs (p. 175-178). Paris : Dunod.

Veil, C. (2012). Vulnérabilités au travail. Toulouse, France : Érès.

Vion, R. (2005). Modalités, modalisations, interaction et dialogisme. In J. Bres (Ed.). Dialogisme et polyphonie. Approches linguistiques (p. 143-156). Louvain-la-Neuve, Belgique : De Boeck Supérieur.

Viviers, S. (2017). Entre défenses et défenses : quelle créativité pour quel métier et quelle santé ? In G. Amado, J.P. Bouilloud, D. Lhuilier, \& A.L. Ulmann (Eds.), La créativité au travail (p. 217-238). Toulouse, France : Érès. 
Vygotski, L. (1925-1932/2017). Conscience, inconscient, émotions. Paris, France : La Dispute.

Vygotski, L. (1934/1997). Pensée et Langage. Paris, France : La Dispute.

\section{RÉSUMÉS}

Cette thèse porte sur l'engagement et le désengagement au travail, qu'elle propose de conceptualiser comme des dynamiques singulières et parfois paradoxales de mobilisation de soi dans l'activité, liées au contexte organisationnel et institutionnel dans lequel elles se déploient, et pouvant varier en fonction des moments, des situations et des personnes.

INDEX

Mots-clés : engagement au travail, désengagement au travail, cliniques du travail, santé au travail, psychopathologie du travail, encadrement intermédiaire, service public, propreté

\section{AUTEURS}

\section{AMÉLIE SANDOVAL}

Conservatoire national des arts et métiers, CRTD, 41 rue Gay Lussac, 75005 Paris amelie.sandoval@gmail.com 